\title{
Effects of protonation and hydrogen bonding on carbon-13 chemical shifts of compounds containing the $\mathrm{C}=\ddot{\mathrm{N}}-$ group $^{1,2}$
}

\author{
Malwina Allen ${ }^{3}$ ANd John D. Roberts ${ }^{4}$ \\ The Gates and Crellin Laboratories of Chemistry, California Institute of Technology, Pasadena, CA 91125, U.S.A. \\ Received March 4, 1980 \\ This paper is dedicated to Prof. Raymond U. Lemieux on the occasion of his 60 th birthday
}

\begin{abstract}
Malwina Allen and John D. Roberts. Can. J. Chem. 59, 451 (1981).
The effects of hydrogen bonding and protonation produced by changing solvent from chloroform to 2,2,2-trifluoroethanol to trifluoroacetic acid were determined for the ${ }^{13} \mathrm{C}$ chemical shifts of fourteen imines, four oximes, and two pyridines. Downfield shifts relative to chloroform were observed for the $\mathrm{C}=\mathrm{N}$ carbon of the imines and oximes in both trifluoroethanol and trifluoroacetic acid, ranging from 1.5 to $27.5 \mathrm{ppm}$, which contrasts with upfield $\mathrm{C}_{\alpha}$ shifts for the pyridines in trifluoroacetic acid. Substantial downfield and upfield shifts of from +10 to $-15 \mathrm{ppm}$ were found for the para and ipso carbons, respectively, of the aromatic rings attached to the $\mathrm{C}=\mathrm{N}$ group in trifluoroacetic acid. Possible explanations of these shift changes are considered as well as their relation to the corresponding changes in ${ }^{15} \mathrm{~N}$ shifts with the identical variations in solvent.
\end{abstract}

Malwina Allen et John D. Roberts. Can. J. Chem. 59, 451 (1981).

Utilisant le chloroforme, la trifluoro-2,2,2 éthanol et l'acide trifluoroacétique comme solvants, on a déterminé les effets de la liaison hydrogène et de la protonation résultant du changement de solvant sur les déplacements chimiques du ${ }^{13} \mathrm{C}$ de 14 imines, de 4 oximes et de deux pyridines. On a observé des déplacements chimiques vers les champs faibles (allant de 1,5 à 27,5 ppm) du carbone $\mathrm{C}=\mathrm{N}$ des imines et des oximes dans le trifluoroéthanol et dans l'acide trifluoroacétique; ces résultats contrastent avec les déplacements chimiques vers les champs forts observés pour les $C_{\alpha}$ des pyridines dans l'acide trifluoroacétique. Dans l'acide trifluoroacétique, on a observé des déplacements importants vers les champs faibles et vers les champs forts allant de $+10 \mathrm{a}-15 \mathrm{ppm}$ pour les carbones respectivement en positions para et ipso des cycles aromatiques liés au groupe $\mathrm{C}=\mathrm{N}$.

On considère des explications probables de ces déplacements aussi bien que leur relation avec les changements correspondants dans les déplacements chimiques du ${ }^{15} \mathrm{~N}$ avec des changements identiques de solvants.

[Traduit par le journal]

\section{Introduction}

In the preceding paper (1), we reported the changes in ${ }^{15} \mathrm{~N}$ chemical shifts associated with the sequence of solvent changes, chloroform $\rightarrow$ 2,2,2-trifluoroethanol $\rightarrow$ trifluoroacetic acid for a number of imines, oximes, and pyridines. The corresponding changes in ${ }^{13} \mathrm{C}$ chemical shifts are discussed here.

Previous studies of the protonation of azaaromatics (2), amino acids (3), aliphatic amines (4), benzenamine (5), and nitriles (6) have demonstrated a general trend of upfield ${ }^{13} \mathrm{C}$ shifts, ranging from 1 to about $17 \mathrm{ppm}$, for the carbons directly attached to the nitrogens. Because imines, oximes, azaaromatics, and nitriles all have multiple bonds between nitrogen and carbon, and because the ${ }^{15} \mathrm{~N}$ shifts of all of the substances move strongly upfield on protonation, it was of interest to determine whether the

${ }^{1}$ Supported by the National Science Foundation, and by the Public Health Service, Research Grant No. GM-11072 from the Division of General Medical Sciences.

${ }^{2}$ Contribution No. 6180

${ }^{3}$ On sabbatical leave from Framingham State College, Framingham, MA 01701, U.S.A.

${ }^{4}$ To whom all correspondence should be addressed.
${ }^{13} \mathrm{C}$ shifts of the carbons multiply bonded to the nitrogens change in a similarly uniform way.

\section{Experimental Section}

The compounds used were the same as in the earlier investigation (1). The ${ }^{13} \mathrm{C}$ spectra of 4 mol\% solutions were taken with a Varian XL-100-15 spectrometer operating at $25.4 \mathrm{MHz}$ in the

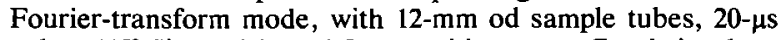
pulses ( $45^{\circ}$ flip angle), and 5-s repetition rates. Good signal-tonoise ratios could usually be obtained for all of the carbons with 200-300 scans. Tetramethylsilane (TMS) was used as internal reference, and a 5 -mm coaxial tube containing deuterium oxide provided the external field frequency lock signal. The samples were at the ambient probe temperature, about $28^{\circ} \mathrm{C}$. The data length was 8192 points for a spectral width of $5000 \mathrm{~Hz}$. The reproducibility of the shifts was about $\pm 0.05 \mathrm{ppm}$.

\section{Results and Discussion}

The ${ }^{13} \mathrm{C}$ chemical shifts of all of the compounds studied in this work in chloroform solution are summarized in Tables 1 to 4 , wherein the compounds are grouped in accord with common structural features. Values for the shifts of some of these compounds have been reported previously (2, 7-9), but were redetermined to ensure constant experimental conditions. The assignments of resonances to particular carbons were generally 
TABLE $1 .{ }^{13} \mathrm{C}$ chemical shifts of $\mathrm{N}$-benzylideneanilines in chloroform

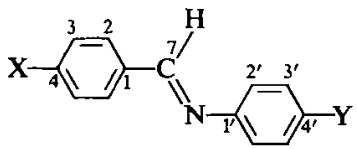

\begin{tabular}{|c|c|c|c|c|c|c|c|c|c|c|c|c|}
\hline \multirow[b]{2}{*}{ Compound } & \multirow[b]{2}{*}{$\mathrm{X}$} & \multirow[b]{2}{*}{$\mathbf{Y}$} & \multicolumn{10}{|c|}{$\delta$, ppm from internal TMS } \\
\hline & & & $\mathrm{C} 7$ & $\mathrm{Cl}$ & $\mathrm{C} 2$ & $\mathrm{C} 3$ & $\mathrm{C} 4$ & $\mathrm{Cl}^{\prime}$ & $\mathrm{C} 2^{\prime}$ & $\mathrm{C}^{\prime}$ & $\mathrm{C}^{\prime}$ & $\mathrm{C} 8^{a}$ \\
\hline $1 a$ & $\mathrm{CH}_{3} \mathrm{O}$ & $\mathrm{H}$ & 159.3 & 129.1 & 130.3 & 114.0 & 162.0 & 152.2 & 120.7 & 128.9 & 125.3 & 55.2 \\
\hline $1 b$ & $\mathrm{CH}_{3}$ & $\mathrm{H}$ & 160.0 & 133.6 & 129.6 & 129.3 & 141.6 & 152.1 & 120.7 & 128.9 & 125.3 & 21.6 \\
\hline $1 c$ & $\mathrm{H}$ & $\mathbf{H}$ & 160.1 & 136.0 & 128.5 & 128.5 & 131.1 & 151.8 & 120.6 & 129.0 & 125.7 & \\
\hline $1 d$ & $\mathrm{O}_{2} \mathrm{~N}$ & $\mathrm{H}$ & 157.0 & 141.4 & 129.2 & 123.8 & 149.1 & 150.7 & 120.8 & 129.2 & 126.9 & \\
\hline $1 e$ & $\mathrm{H}$ & $\mathrm{OCH}_{3}$ & 158.0 & 136.3 & 128.5 & 128.5 & 130.8 & 144.7 & 122.0 & 114.3 & 158.0 & 55.0 \\
\hline $\mathbf{1} f$ & $\mathbf{H}$ & $\mathrm{NO}_{2}$ & 162.4 & 135.3 & 128.8 & 129.1 & 132.2 & 157.7 & 121.1 & 124.8 & 145.3 & \\
\hline
\end{tabular}

TABLE $2 .{ }^{13} \mathrm{C}$ chemical shifts of $N$-benzylidenealkylamines in chloroform

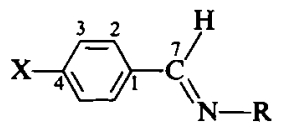

\begin{tabular}{|c|c|c|c|c|c|c|c|c|c|c|c|c|}
\hline \multirow[b]{2}{*}{ Compound } & \multirow[b]{2}{*}{$\mathrm{x}$} & \multirow[b]{2}{*}{$\mathbf{R}$} & \multicolumn{10}{|c|}{$\delta$, ppm from internal TMS } \\
\hline & & & $\mathrm{C} 7$ & $\mathrm{C} 1$ & $\mathrm{C} 2$ & $\mathrm{C} 3$ & $\mathrm{C} 4$ & $\mathrm{Cl}^{\prime}$ & $\mathrm{C} 2^{\prime}$ & $\mathrm{C3}^{\prime}$ & $\mathrm{C}^{\prime}$ & $\mathrm{C} 8$ \\
\hline $2 a$ & $\mathrm{CH}_{3} \mathrm{O}$ & & 157.9 & 129.4 & 129.4 & 114.1 & 161.2 & 69.9 & 25.7 & 34.5 & 25.7 & 55.1 \\
\hline $2 b$ & $\mathrm{O}_{2} \mathrm{~N}$ & & 155.9 & 142.0 & 128.5 & 123.6 & 148.6 & 70.6 & 24.6 & 34.2 & 25.6 & \\
\hline $2 c$ & $\mathbf{H}$ & $\stackrel{\mathrm{I}^{\prime}}{\mathrm{C}} \mathrm{H}\left(\mathrm{CH}_{3}\right)_{2}$ & 158.0 & 136.4 & 127.9 & 128.3 & 130.2 & 61.7 & 24.2 & & & \\
\hline $2 d$ & $\mathbf{H}$ & $\stackrel{\mathrm{I}}{\mathrm{C}} \mathrm{H}_{2} \mathrm{CH}\left(\mathrm{CH}_{3}\right)_{2}$ & 160.5 & 136.3 & 127.9 & 128.3 & 130.4 & 69.7 & 29.6 & 20.7 & & \\
\hline $2 e$ & $\mathbf{H}$ & $\stackrel{\mathbf{I}^{\prime}}{\mathrm{C}} \mathrm{H}_{2} \mathrm{CH}_{3}$ & 160.2 & 136.2 & 127.8 & 128.4 & 130.3 & 55.8 & 16.3 & & & \\
\hline
\end{tabular}

confirmed by single-frequency off-resonance decoupling, relative peak intensities, and comparisons with shift assignments already made for appropriately substituted benzene derivatives. For the imine, $3 a$, the phenyl rings attached to the $\mathrm{C}=\mathrm{N}$ carbon are nonequivalent; the set of resonances similar to those of $3 b$ was assigned to the anti phenyl carbons. This assumes the anti configuration of $3 b$ suggested by Yoshida et al. (9) is correct. The resonances of $3 c$ and $3 d$ were made by analogy with those of the corresponding oximes (7).

The ${ }^{13} \mathrm{C}$ shifts of the imines of Table 1 and of the oximes of Table 4 have been discussed previously $(7,8)$. The $\mathrm{C}=\mathrm{N}$ carbons of the imines in Table 2 are very similar to those of Table 1 , and are rather insensitive to substituents, 4-nitro vs. 4-methoxy, in $2 a-2 b$, and to the various alkyl groups in $2 c-2 e .5$

\footnotetext{
${ }^{s}$ The insensitivity of imines to substituents on adjacent rings is similar to that reported for acetophenones $(10 a)$, styrenes $(10 b)$, and chalconnes $(10 c)$.
}

The resonances of the $\mathrm{C}=\mathrm{N}$ carbons of imines listed in Table 3 are farther downfield than those of Tables 1 and 2 (analogous to ketones vs. aldehydes) with deshielding increasing with the number of the aliphatic groups. Perhaps surprisingly, the change in ring size from six to five between $3 c$ and $3 d$ causes as large a shift change as going from $3 a$ to $3 c$.

The changes in ${ }^{13} \mathrm{C}$ shifts $(\Delta \delta)$ which occur on changing the solvent from chloroform to trifluoroethanol and from chloroform to trifluoroacetic acid are listed in Tables 5 to $9 .^{6}$ The assignments of the resonances of the $2^{\prime}$ and $3^{\prime}$ carbons of $1 a-1 f$ in trifluoroacetic acid and of the aliphatic carbons of $3 c, 3 d$, and $4 b$, in trifluoroacetic acid are tentative, as therefore will be the $\Delta \delta$ values for these substances. Concentration effects on the ${ }^{13} \mathrm{C}$ resonances of representative compounds in the 2 , 4,8 , and $10 \mathrm{~mol} \%$ range were within $0.1 \mathrm{ppm}$ (more

${ }^{6}$ The reasons for choosing chloroform as the standard solvent were discussed earlier (1). 
TABLE $3 .{ }^{13} \mathrm{C}$ chemical shifts of ketimines in chloroform

\begin{tabular}{|c|c|c|c|c|c|c|c|c|c|c|c|c|c|c|c|}
\hline & \multirow[b]{2}{*}{ Compound } & \multicolumn{13}{|c|}{$\delta$, ppm from internal TMS } & \multirow[b]{2}{*}{$\mathrm{C} 8$} \\
\hline & & $\mathrm{C} 7$ & $\mathrm{C} 1$ & $\mathrm{C} 2$ & $\mathrm{C} 3$ & $\mathrm{C} 4$ & $\mathrm{C} 5$ & C6 & $\mathrm{Cl}^{\prime}$ & $\mathrm{C} 2^{\prime}$ & $\mathrm{C}^{\prime}$ & $\mathrm{C}^{\prime}$ & $\mathrm{C1}^{\prime \prime}$ & $\mathrm{C} 4^{\prime \prime}$ & \\
\hline $3 a$ & & 167.8 & 139.5 & $a$ & $a$ & 130.5 & & & 151.2 & $a$ & $a$ & 122.9 & 136.1 & 128.3 & \\
\hline $3 b$ & & 165.2 & 139.4 & 127.1 & 128.2 & 130.3 & & & 151.6 & 119.3 & 128.8 & 123.1 & & & 17.3 \\
\hline $3 c$ & & & 174.6 & 27.8 & 27.6 & 25.9 & 31.2 & 39.4 & 150.6 & 119.7 & 128.6 & 122.8 & & & \\
\hline $3 d$ & & & 182.1 & 30.6 & 24.9 & 24.1 & 36.2 & & 152.0 & 119.0 & 128.9 & 123.0 & & & \\
\hline
\end{tabular}

TABLE $4 .{ }^{13} \mathrm{C}$ chemical shifts of oximes in chloroform

\begin{tabular}{|c|c|c|c|c|c|c|c|c|c|}
\hline & \multirow[b]{2}{*}{ Compound } & \multicolumn{8}{|c|}{$\delta$, ppm from internal TMS } \\
\hline & & $\mathrm{C} 7$ & $\mathrm{Cl}$ & $\mathrm{C} 2$ & $\mathrm{C} 3$ & $\mathrm{C} 4$ & $\mathrm{C} 5$ & $\mathrm{C} 6$ & $\mathrm{C} 8$ \\
\hline $4 a^{a}$ & $\begin{array}{r}3 \\
8 \\
\mathrm{H}_{3} \mathrm{C}\end{array}$ & 150.3 & 129.2 & 129.5 & 127.0 & 140.2 & & & 21.3 \\
\hline $4 b$ & & & 147.6 & 11.2 & & & & & \\
\hline $4 c^{b}$ & & & 147.9 & 15.0 & & & & & \\
\hline $4 d^{b}$ & & & 160.3 & 25.8 & 25.6 & 24.4 & 26.7 & 31.9 & \\
\hline
\end{tabular}

${ }^{a}$ Configuration as given in ref. 8 .

${ }^{b} \mathrm{~A}$ mixture of $4 b$ and $4 c$, consisting mostly of one isomer, was used. The major signals were attributed to $4 b$ on the basis of ref. 8.

substantial changes were noted at $20 \mathrm{~mol} \%$ ) and it appears that the shifts at infinite dilution are already well approximated by those at the $10 \mathrm{~mol} \%$ level.

It will be seen from Tables 5 to 9 that changing from chloroform to either trifluoroethanol or trifluoroacetic acid produces substantial changes in many of the carbon chemical shifts of the compounds studied. Both upfield and downfield shift changes are observed, and it is especially interesting and significant that trifluoroethanol and trifluoroacetic acid may have effects which are not only different in magnitude but in sign as well. It is not wholly surprising that this be the case, because, as we have pointed out when discussing the ${ }^{15} \mathrm{~N}$ shifts (1), trifluoroethanol is primarily a hydrogenbonding solvent, while trifluoroacetic acid is a protonating solvent for imines. Protonation with trifluoroacetic acid is firmly established by observation of proton- ${ }^{15} \mathrm{~N}$ couplings with imines (1).

For the ${ }^{15} \mathrm{~N}$ resonances of $\mathrm{C}=\mathrm{N}$ nitrogens, both 
TABLE 5. Effects of hydrogen bonding and protonation on ${ }^{13} \mathrm{C}$ chemical shifts of $N$-benzylideneanilines

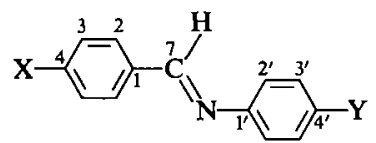

\begin{tabular}{|c|c|c|c|c|c|c|c|c|c|c|c|c|c|}
\hline \multirow[b]{2}{*}{ Compound } & \multirow[b]{2}{*}{$\mathrm{X}$} & \multirow[b]{2}{*}{$\mathrm{Y}$} & \multirow[b]{2}{*}{ Solvent ${ }^{a}$} & \multicolumn{10}{|c|}{$\Delta \delta^{b}=\left(\delta_{\text {solvent }}-\delta_{\mathrm{CHCl}_{3}}\right), \mathrm{ppm}$} \\
\hline & & & & $\mathrm{C} 7$ & $\mathrm{C} 1$ & $\mathrm{C} 2$ & $\mathrm{C} 3$ & $\mathrm{C} 4$ & $\mathrm{C} 1^{\prime}$ & $\left(\mathrm{C} 2^{\prime}\right.$ & $\left(3^{\prime}\right)^{c}$ & $\mathrm{C}^{\prime}$ & $\mathrm{C} 8^{d}$ \\
\hline $1 a$ & $\mathrm{CH}_{3} \mathrm{O}$ & $\mathrm{H}$ & $\begin{array}{l}\text { TFE } \\
\text { TFA }\end{array}$ & $\begin{array}{l}5.1 \\
5.4\end{array}$ & $\begin{array}{l}-0.2 \\
-8.6\end{array}$ & $\begin{array}{l}2.3 \\
7.3\end{array}$ & $\begin{array}{l}1.7 \\
4.1\end{array}$ & $\begin{array}{l}2.7 \\
9.7\end{array}$ & $\begin{array}{r}0.1 \\
-14.4\end{array}$ & $\begin{array}{l}1.5 \\
0.9\end{array}$ & $\begin{array}{l}1.8 \\
3.1\end{array}$ & $\begin{array}{l}2.5 \\
7.3\end{array}$ & $\begin{array}{l}0.9 \\
2.1\end{array}$ \\
\hline $\mathbf{1} b$ & $\mathrm{CH}_{3}$ & $\mathrm{H}$ & $\begin{array}{l}\text { TFE } \\
\text { TFA }\end{array}$ & $\begin{array}{l}5.2 \\
6.9\end{array}$ & $\begin{array}{l}-0.1 \\
-8.4\end{array}$ & $\begin{array}{l}1.6 \\
5.3\end{array}$ & $\begin{array}{l}1.6 \\
4.3\end{array}$ & $\begin{array}{r}3.2 \\
14.4\end{array}$ & $\begin{array}{r}0.0 \\
-15.0\end{array}$ & $\begin{array}{l}1.3 \\
1.3\end{array}$ & $\begin{array}{l}1.7 \\
3.1\end{array}$ & $\begin{array}{l}2.2 \\
6.6\end{array}$ & $\begin{array}{l}0.0 \\
1.4\end{array}$ \\
\hline $1 c$ & $\mathbf{H}$ & $\mathbf{H}$ & $\begin{array}{l}\text { TFE } \\
\text { TFA } \\
\text { HOAC }\end{array}$ & $\begin{array}{l}5.3 \\
7.7 \\
2.7\end{array}$ & $\begin{array}{r}0.2 \\
-8.2 \\
-2.8\end{array}$ & $\begin{array}{l}1.6 \\
5.5 \\
1.1\end{array}$ & $\begin{array}{l}1.6 \\
3.1 \\
0.9\end{array}$ & $\begin{array}{r}2.4 \\
10.1 \\
4.4\end{array}$ & $\begin{array}{r}0.2 \\
-\underset{e}{14.5}\end{array}$ & $\begin{array}{l}1.6 \\
1.4 \\
1.2\end{array}$ & $\begin{array}{l}1.5 \\
3.6 \\
0.9\end{array}$ & $\begin{array}{l}2.3 \\
7.9 \\
1.5\end{array}$ & \\
\hline $1 d$ & $\mathrm{O}_{2} \mathrm{~N}$ & $\mathrm{H}$ & $\begin{array}{l}\text { TFE } \\
\text { TFA }\end{array}$ & $\begin{array}{l}5.1 \\
9.0\end{array}$ & $\begin{array}{r}1.1 \\
-8.1\end{array}$ & $\begin{array}{l}1.8 \\
6.1\end{array}$ & $\begin{array}{l}1.5 \\
3.0\end{array}$ & $\begin{array}{l}1.8 \\
5.5\end{array}$ & $\begin{array}{r}1.0 \\
-13.4\end{array}$ & $\begin{array}{l}1.2 \\
2.0\end{array}$ & $\begin{array}{l}1.8 \\
3.4\end{array}$ & $\begin{array}{l}2.1 \\
8.2\end{array}$ & \\
\hline $1 e$ & $\mathbf{H}$ & OCH & $\begin{array}{l}\text { TFE } \\
\text { TFA }\end{array}$ & $\begin{array}{l}5.6 \\
7.1\end{array}$ & $\begin{array}{r}0.1 \\
-8.2\end{array}$ & $\begin{array}{l}1.5 \\
3.6\end{array}$ & $\begin{array}{l}1.6 \\
2.5\end{array}$ & $\begin{array}{r}3.4 \\
10.0\end{array}$ & $\begin{array}{r}0.6 \\
-13.7\end{array}$ & $\begin{array}{l}1.4 \\
1.9\end{array}$ & $\begin{array}{l}1.7 \\
3.3\end{array}$ & $\begin{array}{l}1.9 \\
6.0\end{array}$ & $\begin{array}{l}1.0 \\
1.7\end{array}$ \\
\hline $\mathbf{1} f$ & $\mathbf{H}$ & $\mathrm{NO}_{2}$ & $\begin{array}{l}\text { TFE }^{f} \\
\text { TFA }\end{array}$ & $\overline{9.2}$ & $-\overline{7.6}$ & $\overline{6.4}$ & $\overline{3.7}$ & $\overline{10.9}$ & $-\overline{15.1}$ & $\overline{3.0}$ & $\overline{2.9}$ & $\overline{5.2}$ & - \\
\hline
\end{tabular}

${ }^{a} \mathrm{TFE}=\mathrm{CF}_{3} \mathrm{CH}_{2} \mathrm{OH}, \mathrm{TFA}=\mathrm{CF}_{3} \mathrm{CO}_{2} \mathrm{H}, \mathrm{HOAC}=\mathrm{CH}_{3} \mathrm{CO}_{2} \mathrm{H}$.

Positive signs signify downfield shifts, negative signs upfield shifts.

${ }^{6 T h e}{ }^{13} \mathrm{C}$ assignments for these atoms are tentative, and perhaps larger solvent changes should be expected for $\mathrm{C}^{\prime}$ than for $\mathrm{C} 3{ }^{\prime}$. $\mathrm{However}$, other possible assignments gave $\Delta \delta$ 's which were less consistent and, in fact, unreasonable in some instances.

The substituent carbon.

Signal not seen in the spectrum.

sCompound not sufficiently soluble to obtain a spectrum.

TABLE 6. Effects of hydrogen bonding and protonation on ${ }^{13} \mathrm{C}$ chemical shifts of $N$-benzylidenealkylamines

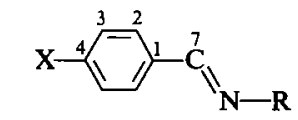

\begin{tabular}{|c|c|c|c|c|c|c|c|c|c|c|c|c|c|}
\hline \multirow[b]{2}{*}{ Compound } & \multirow[b]{2}{*}{$x-$} & \multirow[b]{2}{*}{$-\mathbf{R}$} & \multirow[b]{2}{*}{ Solvent ${ }^{a}$} & \multicolumn{10}{|c|}{$\Delta \delta^{a}=\left(\delta_{\text {solvent }}-\delta_{\mathrm{CHCl}_{3}}\right), \mathrm{ppm}$} \\
\hline & & & & $\mathrm{C} 7$ & $\mathrm{C} 1$ & $\mathrm{C} 2$ & $\mathrm{C} 3$ & $\mathrm{C} 4$ & $\mathrm{Cl}^{\prime}$ & $\mathrm{C} 2^{\prime}$ & $\mathrm{C}^{\prime}$ & $\mathrm{C}^{\prime}$ & $\mathrm{C} 8$ \\
\hline $2 a$ & $\mathrm{CH}_{3} \mathrm{O}-$ & & $\begin{array}{l}\text { TFE } \\
\text { TFA }\end{array}$ & $\begin{array}{r}5.4 \\
10.7\end{array}$ & $\begin{array}{l}-0.1 \\
-9.1\end{array}$ & $\begin{array}{l}2.1 \\
7.1\end{array}$ & $\begin{array}{l}1.3 \\
3.7\end{array}$ & $\begin{array}{l}2.5 \\
9.6\end{array}$ & $\begin{array}{r}1.4 \\
-4.3\end{array}$ & $\begin{array}{l}0.5 \\
0.1\end{array}$ & $\begin{array}{r}0.7 \\
-0.8\end{array}$ & $\begin{array}{l}0.5 \\
0.1\end{array}$ & $\begin{array}{l}0.9 \\
2.0\end{array}$ \\
\hline $2 b$ & $\mathrm{O}_{2} \mathrm{~N}-$ & & $\begin{array}{l}\text { TFE } \\
\text { TFA }\end{array}$ & $\begin{array}{r}5.8 \\
14.4\end{array}$ & $\begin{array}{r}0.5 \\
-9.1\end{array}$ & $\begin{array}{l}1.9 \\
6.1\end{array}$ & $\begin{array}{l}1.5 \\
6.0\end{array}$ & $\begin{array}{l}1.9 \\
5.7\end{array}$ & $\begin{array}{r}2.0 \\
-2.8\end{array}$ & $\begin{array}{l}1.3 \\
1.1\end{array}$ & $\begin{array}{r}0.8 \\
-1.0\end{array}$ & $\begin{array}{l}0.8 \\
0.1\end{array}$ & \\
\hline $2 c$ & $\mathbf{H}-$ & $-\stackrel{\mathrm{I}^{\prime}}{\mathrm{C}} \mathrm{H}\left(\mathrm{CH}_{3}\right)_{2}$ & $\begin{array}{l}\text { TFE } \\
\text { TFA }\end{array}$ & $\begin{array}{r}5.3 \\
13.3\end{array}$ & $\begin{array}{l}-0.2 \\
-9.8\end{array}$ & $\begin{array}{l}2.0 \\
5.3\end{array}$ & $\begin{array}{l}1.9 \\
3.6\end{array}$ & $\begin{array}{r}2.4 \\
10.5\end{array}$ & $\begin{array}{r}1.3 \\
-1.9\end{array}$ & $\begin{array}{l}-0.3 \\
-2.3\end{array}$ & & & \\
\hline $2 d$ & $\mathrm{H}-$ & $-\stackrel{I^{\circ}}{\mathrm{C}} \mathrm{H}_{2} \stackrel{2^{\prime}}{\mathrm{C}} \mathrm{H}\left(\mathrm{CH}_{3}\right)_{2}$ & $\begin{array}{l}\text { TFE } \\
\text { TFA } \\
\text { HOAc }\end{array}$ & $\begin{array}{r}5.8 \\
13.5 \\
12.1\end{array}$ & $\begin{array}{l}-0.2 \\
-9.0 \\
-7.7\end{array}$ & $\begin{array}{l}1.6 \\
5.3 \\
5.2\end{array}$ & $\begin{array}{l}1.7 \\
3.7 \\
2.5\end{array}$ & $\begin{array}{r}2.5 \\
10.6 \\
7.8\end{array}$ & $\begin{array}{r}0.3 \\
-7.3 \\
-7.9\end{array}$ & $\begin{array}{r}0.8 \\
0.8 \\
-0.5\end{array}$ & $\begin{array}{r}0.0 \\
-1.0 \\
b\end{array}$ & & \\
\hline $2 e$ & $\mathrm{H}-$ & $-\stackrel{1^{\prime}}{\mathrm{C}} \mathrm{H}_{2} \stackrel{2^{\prime}}{\mathrm{C}} \mathrm{H}_{3}$ & TFA & 13.1 & -8.8 & 5.3 & 3.5 & 10.3 & -5.0 & -2.3 & & & \\
\hline
\end{tabular}

See Table 5 .

O Overlapped by $\mathrm{CH}_{3} \mathrm{CO}_{2} \mathrm{H}$ resonance.

trifluoroacetic acid and trifluoroethanol uniformly cause upfield shifts, with the protonation shifts being several times the hydrogen-bonding shifts. These shift changes accord with the expected increases in the $n \rightarrow \pi *$ transition energies, which are associated with very substantial second-order paramagnetic contributions to the chemical shifts of $-\mathrm{C}=\ddot{\mathrm{N}}$ - nitrogens. This does not mean to say that other effects on the ${ }^{15} \mathrm{~N}$ chemical shifts are negligible, only that they appear to be largely 
TABLE 7. Effects of hydrogen bonding and protonation on ${ }^{13} \mathrm{C}$ chemical shifts of ketimines

\begin{tabular}{|c|c|c|c|c|c|c|c|c|c|c|c|c|c|c|c|}
\hline \multirow[b]{2}{*}{ Compound } & \multirow[b]{2}{*}{ Solvent $t^{a}$} & \multicolumn{14}{|c|}{$\Delta \delta^{a}=\delta_{\text {solvent }}-\delta_{\mathrm{CHCl}_{3}}, \mathrm{ppm}$} \\
\hline & & $\mathrm{C} 7$ & $\mathrm{C} 1$ & $\mathrm{C} 2$ & $\mathrm{C} 3$ & $\mathrm{C} 4$ & $\mathrm{C} 5$ & C6 & $\mathrm{C}^{\prime}$ & $\mathrm{C} 2^{\prime}$ & $\mathrm{C} 3^{\prime}$ & $\mathrm{C}^{\prime}$ & $\mathrm{C} 1^{\prime \prime}$ & $\mathrm{C} 4^{\prime \prime}$ & $\mathrm{C} 8$ \\
\hline $3 a$ & $\begin{array}{l}\text { TFE } \\
\text { TFA }\end{array}$ & $\begin{array}{r}6.8 \\
16.0\end{array}$ & $\begin{array}{r}1.5 \\
-5.3\end{array}$ & $a$ & $\begin{array}{l}b \\
b\end{array}$ & $\begin{array}{l}1.8 \\
8.9\end{array}$ & & & $\begin{array}{r}-0.2 \\
-14.0\end{array}$ & $\begin{array}{l}b \\
b\end{array}$ & $\begin{array}{l}b \\
b\end{array}$ & $\begin{array}{l}1.8 \\
9.4\end{array}$ & $\begin{array}{r}1.0 \\
-5.5\end{array}$ & $\begin{array}{l}2.1 \\
8.8\end{array}$ & \\
\hline $3 b$ & $\begin{array}{l}\text { TFE } \\
\text { TFA }\end{array}$ & $\begin{array}{r}7.8 \\
23.5\end{array}$ & $\begin{array}{r}1.7 \\
-6.9\end{array}$ & $\begin{array}{l}1.3 \\
5.0\end{array}$ & $\begin{array}{l}1.8 \\
2.0\end{array}$ & $\begin{array}{l}2.1 \\
9.8\end{array}$ & & & $\begin{array}{r}-0.6 \\
-15.5\end{array}$ & $\begin{array}{l}2.1 \\
6.6\end{array}$ & $\begin{array}{l}1.9 \\
3.1\end{array}$ & $\begin{array}{r}3.1 \\
10.1\end{array}$ & & & $\begin{array}{l}2.1 \\
3.4\end{array}$ \\
\hline $3 c$ & $\begin{array}{l}\text { TFE } \\
\text { TFA } \\
\text { HOAc }\end{array}$ & & $\begin{array}{r}7.5 \\
27.5 \\
19.1\end{array}$ & $\begin{array}{l}1.2 \\
(2.5 \\
(0.1\end{array}$ & $\begin{array}{l}1.2 \\
2.5 \\
0.0\end{array}$ & $\begin{array}{r}0.7 \\
-0.5 \\
-0.8\end{array}$ & $\begin{array}{r}1.6 \\
2.5 \\
-3.3\end{array}$ & $\begin{array}{c}0.1 \\
-0.6)^{c} \\
2.7)^{c}\end{array}$ & $\begin{array}{r}-0.9 \\
-15.9 \\
-11.6\end{array}$ & $\begin{array}{l}2.2 \\
5.6 \\
5.4\end{array}$ & $\begin{array}{l}1.9 \\
5.6 \\
1.8\end{array}$ & $\begin{array}{r}3.0 \\
10.0 \\
6.9\end{array}$ & & & \\
\hline $3 d$ & $\begin{array}{l}\text { TFE } \\
\text { TFA }\end{array}$ & & $\begin{array}{r}7.3 \\
26.7\end{array}$ & $\begin{array}{r}1.8 \\
(6.0\end{array}$ & $\begin{array}{l}0.7 \\
1.1\end{array}$ & $\begin{array}{l}1.0 \\
1.1\end{array}$ & $\begin{array}{l}0.6 \\
2.3)^{c}\end{array}$ & & $\begin{array}{r}-1.1 \\
-15.4\end{array}$ & $\begin{array}{l}2.0 \\
4.6\end{array}$ & $\begin{array}{l}1.5 \\
3.9\end{array}$ & $\begin{array}{l}3.0 \\
8.8\end{array}$ & & & \\
\hline
\end{tabular}

aSee Table 5.

作

cThese values based on tentative assignments.

TABLE 8. Effects of hydrogen bonding and protonation on ${ }^{13} \mathrm{C}$ chemical shifts of oximes

\begin{tabular}{|c|c|c|c|c|c|c|c|c|c|}
\hline \multirow[b]{2}{*}{ Compound } & \multirow[b]{2}{*}{ Solvent ${ }^{a}$} & \multicolumn{8}{|c|}{$\Delta \delta^{a}=\delta_{\text {solvent }}-\delta_{\mathrm{CHCl}_{3}}, \mathrm{ppm}$} \\
\hline & & $\mathrm{C} 7$ & $\mathrm{Cl}$ & $\mathrm{C} 2$ & $\mathrm{C} 3$ & $\mathrm{C} 4$ & C5 & C6 & $\mathrm{C} 8$ \\
\hline $4 a$ & $\begin{array}{l}\text { TFE } \\
\text { TFA }\end{array}$ & $\begin{array}{l}3.5 \\
1.8\end{array}$ & $\begin{array}{r}0.7 \\
-5.9\end{array}$ & $\begin{array}{l}1.5 \\
7.0\end{array}$ & $\begin{array}{l}1.4 \\
5.2\end{array}$ & $\begin{array}{r}2.8 \\
11.5\end{array}$ & & & $\begin{array}{l}0.2 \\
1.2\end{array}$ \\
\hline $4 b^{b}$ & $\begin{array}{l}\text { TFE } \\
\text { TFA }\end{array}$ & & $\begin{array}{r}4.0 \\
11.0\end{array}$ & $\begin{array}{l}0.2 \\
1.9\end{array}$ & & & & & \\
\hline $4 c$ & $\begin{array}{l}\text { TFE } \\
\text { TFA }\end{array}$ & & $\begin{array}{r}4.5 \\
11.3\end{array}$ & $\begin{array}{r}0.2 \\
-1.5\end{array}$ & & & & & \\
\hline $4 d$ & $\begin{array}{l}\text { TFE } \\
\text { TFA } \\
\text { HOAc }\end{array}$ & & $\begin{array}{r}5.9 \\
20.3 \\
1.2\end{array}$ & $\begin{array}{l}(1.0 \\
(2.2 \\
(0.4\end{array}$ & $\begin{array}{l}1.0)^{c} \\
1.5 \\
0.6)^{c}\end{array}$ & $\begin{array}{l}1.5 \\
1.0 \\
1.1\end{array}$ & $\begin{array}{l}1.2 \\
1.7 \\
0.8\end{array}$ & $\begin{array}{l}0.9 \\
1.0)^{c} \\
0.3\end{array}$ & \\
\hline
\end{tabular}

${ }^{a}$ See Table 5 .

${ }^{b} \mathrm{~A}$ mixture of $4 b$ and $4 c$, consisting mostly of $4 b$, was used.

cBased on tentative assignments.

masked by the changes in second-order paramagnetic effects associated with partial or full bonding to the unshared electron pair on nitrogen. Because the carbons with which we are concerned have no unshared pairs, it is to be expected that different considerations are likely to apply in rationalizing the positions of their resonance signals.

In the following discussion, we will give special attention to the ${ }^{13} \mathrm{C}$ shift changes which are associated with hydrogen bonding and protonation of the carbons of the structural units, $\mathrm{C}-\mathrm{C}=\mathrm{N}-\mathrm{C}$ of imines and pyridines, and $\mathrm{C}-\mathrm{C}=\mathrm{N}-\mathrm{OH}$ of oximes. Perusal of Tables 5 to 9 shows that the aromatic carbon resonances, except for those of the ipso carbons, move downfield on both hy- 
TABLE 9. Effects of hydrogen bonding and protonation on ${ }^{13} \mathrm{C}$ chemical shifts of pyridines

\begin{tabular}{|c|c|c|c|c|}
\hline \multirow[b]{2}{*}{ Compound } & \multirow[b]{2}{*}{ Solvent $^{a}$} & \multicolumn{3}{|c|}{$\Delta \delta^{b}=\delta_{\text {solvent }}-\delta_{\mathrm{CHCl}_{3}}, \mathrm{ppm}$} \\
\hline & & $\mathrm{C} 2$ & $\mathrm{C} 3$ & $\mathrm{C} 4$ \\
\hline 5a Pyridine $^{c}$ & $\begin{array}{l}\text { TFE } \\
\text { HOAc } \\
\text { TFA }\end{array}$ & $\begin{array}{l}-0.5 \\
-5.3 \\
-6.9\end{array}$ & $\begin{array}{l}2.3 \\
2.7 \\
5.9\end{array}$ & $\begin{array}{r}3.4 \\
7.0 \\
13.7\end{array}$ \\
\hline $5 b$ 2,6-Dimethylpyridine ${ }^{d}$ & $\begin{array}{l}\text { TFE } \\
\text { TFA }\end{array}$ & $\begin{array}{r}1.7 \\
-1.4\end{array}$ & $\begin{array}{l}3.0 \\
2.6\end{array}$ & $\begin{array}{r}3.6 \\
12.5\end{array}$ \\
\hline
\end{tabular}

drogen bonding and protonation, as befits conversion of the $\mathrm{C}=\mathrm{N}$ groups into more electronattracting groups. The changes in shift of these aromatic carbons are often large, especially on protonation, but there are no surprises in their general trends. The aliphatic carbon-shift changes outside of the structural units, $\mathrm{C}-\mathrm{C}=\mathrm{N}-\mathrm{C}$ or $\mathrm{C}-\mathrm{C}=\mathrm{N}-\mathrm{OH}$, are generally small and somewhat erratic and will not be considered further.

One interesting aspect of the shift changes in Tables 5 to 9 is the fact that hydrogen bonding or protonation uniformly causes the resonances of $\mathrm{C}=\mathrm{N}$ carbons in imines and oximes to move downfield while, in contrast, the resonances of $\mathrm{C} 2,6$ of pyridine move upfield. 2,6-Dimethylpyridine is different from pyridine in that its $\mathrm{C} 2,6$ resonances move downfield on hydrogen bonding and upfield on protonation. Also significant are the large and uniform upfield protonation shifts of aromatic ipso carbons which we shall henceforth designate as $\mathrm{C} 1$ and $\mathrm{C}^{\prime}{ }^{\prime}$ in the structural unit $\mathrm{C}^{1}-\mathrm{C}=\mathrm{N}-\mathrm{C}^{1}$. When $\mathrm{C} 1$ is a saturated carbon, as in $3 b-d$ and $4 b-d$, the corresponding protonation shifts (here, of the carbon actually labeled in the formulas variously as $\mathrm{C} 8, \mathrm{C} 2,5, \mathrm{C} 2,6$, or $\mathrm{C} 2$ ) are medium in size and downfield. However, as with $2 c-e$, when $\mathrm{Cl}^{\prime}$ is aliphatic, the shift changes are medium and upfield.

The hydrogen-bonding shifts of the $\mathrm{C} 1$ and $\mathrm{Cl}^{\prime}$ resonances are generally quite small compared to those of the $\mathrm{C}=\mathrm{N}$ carbon resonances and may or may not have the same sign as the protonation shifts. To summarize (where $\mathrm{C}_{\mathrm{ar}}=$ ipso aromatic ring carbon and $\mathrm{C}_{\mathrm{ali}}=$ saturated carbon):

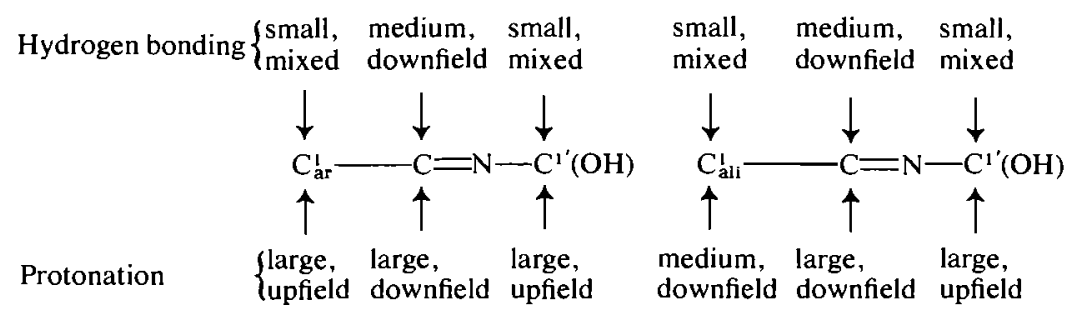

What sense can be made out of these patterns of shift changes? Let us consider the effects on the $\mathrm{C}=\mathrm{N}$ carbon resonances first, remembering that the $\alpha$-carbon resonances of pyridine go upfield on hydrogen bonding or protonation - opposite to the imine and oxime $\mathrm{C}=\mathrm{N}$ resonances.

Partial or complete bonding to the lone pair of a $\mathrm{C}=\mathrm{N}$ nitrogen by a proton donor is expected to raise the $\mathrm{n} \rightarrow \pi *$ transition energy and cause a very substantial upfield shift of the ${ }^{15} \mathrm{~N}$ resonance. Such bonding will also be expected to change the character of the $\mathrm{C}=\mathrm{N}$ bond, as can be rationalized by an increase in the contribution of resonance structures such as $6 b$. (The resonance structures, $\mathbf{6}$, are written for convenience to correspond to full protonation, but the extension to a hydrogenbonding situation should be obvious.)

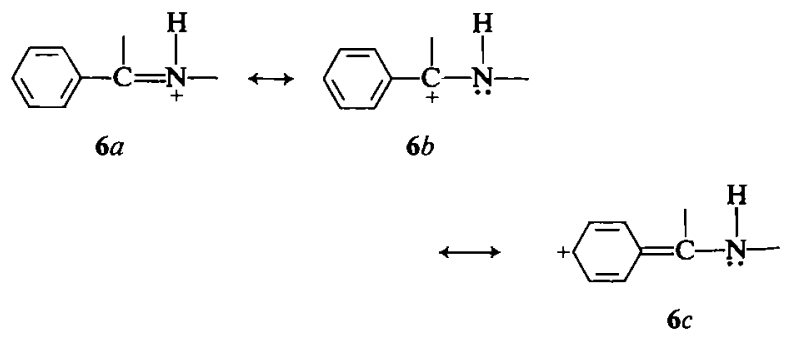


If an aromatic ring is attached to the carbon of the $\mathrm{C}=\mathrm{N}$ bond, then both hydrogen bonding and protonation could be expected to lead to electron delocalization from the ring, as symbolized by $6 c$, and this, of course, is expected to affect the shieldings of the ring carbons in a significant way in the downfield direction, as is observed. An important question is what part, if any, of the shift change at the $\mathrm{C}=\mathrm{N}$ carbon could be a neighboring anisotropy effect arising from the decrease in the paramagnetic shielding of the nitrogen nucleus associated with hydrogen bonding or protonation. Such neighboring anisotropy effects have been used to account for the shielding of acetylenic and non-hydrogen bonded $\mathrm{H}-\mathrm{O}, \mathrm{H}-\mathrm{N}$, and $\mathrm{H}-\mathrm{F}$ protons (11). Whether a change in the electron circulation around the nitrogen should be expected to shield or deshield a directly attached $\mathrm{C}=\mathrm{N}$ carbon nucleus is not easy to predict with molecules as complex as the ones considered here. However, shielding at nitrogen and deshielding at carbon of $\mathrm{C}=\mathrm{N}$ atoms as the result of changing the second-order paramagnetic contributions by hydrogen bonding to, or protonation of, the nitrogen is certainly not unreasonable.

Experimentally, for the compounds in Tables 5 to 8 , the ratios of the changes produced by hydrogen bonding and protonation on the $\mathrm{C}=\mathrm{N}$ carbon resonances are substantially smaller than are the ratios of the corresponding effects on the $\mathrm{C} 4$ resonances of the aromatic carbons. This fact, at least naively, seems to suggest a sizeable contribution to the downfield shift in the $\mathrm{C}=\mathrm{N}$ shifts through the diamagnetic change in the nitrogen shielding as the result of hydrogen bonding. On full protonation, this contribution would be expected to be enhanced, but then might be masked by an opposing shift associated with drastic changes in the overall electron distribution ascribable to resonance structures $6 b$ and $6 c$. These notions are in good agreement with the fact that the shifts of $\mathrm{C} 1$ and $\mathrm{Cl}^{\prime}$ are only very slightly changed by hydrogen bonding but are greatly changed by protonation, as would be expected if there is not in fact much charge transfer on hydrogen bonding. Pyridine and 2,6-dimethylpyridine (Table 9), where the $\mathrm{C}=\mathrm{N}$ atoms are part of aromatic rings, could well be different from imines and oximes because of different anisotropies of the second-order paramagnetic effect at their nitrogen atoms.

The results with compounds $1 a-1 d$ indicate a qualitative relationship between shift changes on protonation and relative contributions of $6 b$ and $6 c$. With $1 a$ and $1 b$, which can enhance the contribution of $6 c$, the $C 4$ shifts are larger and the $C 7$ shifts smaller than for $1 d$ with its 4-nitro group, which should favor $6 b$ over $6 c$. That both the $\mathrm{C} 7$ and $\mathrm{C} 4$ shifts of $1 a$ are smaller than $1 b$ is expected because of electron delocalization from the methoxy group, 7.

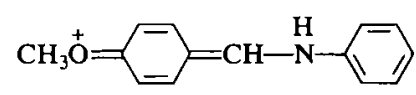

7

The large upfield shifts of the resonances of $\mathrm{C} 1$ and $\mathrm{Cl}^{\prime}$ on protonation have several parallels in compounds where a positive center is generated adjacent to carbon. For example, formation of the conjugate acid of acetophenone with trifluoromethanesulfonic acid causes an upfield shift of the ipso carbon of $8 \mathrm{ppm}$ (5), ionization of triphenylmethanol in sulfuric acid results in an upfield shift of $9.9 \mathrm{ppm}$ for the ipso carbons (12), conversion of benzenamine to its salt with methanesulfonic acid gives an upfield $\mathrm{C} 1$ shift of $17.6 \mathrm{ppm}$ (5). In all of these substances, as with the compounds studied here, the other ring-carbon resonances move downfield. The upfield shift changes produced by attachment of carbon to an atom undergoing a loss of electronic charge seems very likely to be largely an inductive effect. Clearly, conjugative unsaturation is not necessary, because conversion of a carboxylate salt to the acid results in an upfield shift of the carboxyl carbon (13) and the change of an aliphatic amine to the ammonium ion causes an upfield shift of the $\alpha$ carbon (4). The fact that protonation of imines is expected to call for an increase in conjugation involving $\mathrm{Cl}(6 \mathrm{c})$ and a decrease in conjugation involving $\mathrm{C}^{\prime}$ (8) adds further weight to postulation of a nonconjugative origin to the resulting shift changes.

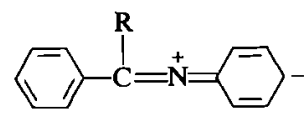

8

It has been pointed out to us by Reynolds that the $\pi$ inductive effect is likely to be especially important in these systems in determining the shifts of the ipso carbons and, furthermore, can help to account for the smaller shift changes of the ortho relative to the para carbons of the unsubstituted phenyl rings of $1 a-1 f, 2 c-2 e$, and $3 b-3 d$ on protonation $(14,15)$.

1. M. Allen and J. D. Roberts. To be published.

2. R. J. Pugmire and D. M. Grant. J. Am. Chem. Soc. 90, 697 (1968).

3. A. R. Quirt, J. R. Lyerla, JR., I. R. Pear, J. S. Cohen, W. F. Reynolds, and M. H. Freedman. J. Am. Chem. Soc. 96, 570 (1974).

4. I. Morishima, K. Yoshikawa, K. OKadA, T. 
Yonezawa, and K. Goto. J. Am. Chem. Soc. 95, 165 (1973).

5. G. L. Nelson, G. C. Levy, and J. D. Cargiolo. J. Am. Chem. Soc. 94, 3089 (1972).

6. G. A. Olah and T. E. Kiovsky. J. Am. Chem. Soc. 90, 4666 (1968).

7. G. E. Hawkes, K. Herwig, and J. D. Roberts. J. Org. Chem. 39, 1017 (1974).

8. N. Inamoto, K. Kushida, S. Masuda, H. Ohta, S. Satoh, Y. TAmura, K. Tokumaru, K. Tori, and M. YoshidA. Tetrahedron Lett. 41, 3617 (1974).

9. M. Yoshida, H. Minato, and M. Kobayashi. Chem. Lett. 1097 (1976).
10. (a) K. S. Dhamı and J. B. Stothers. Can. J. Chem. 43, 479 (1965); (b) K. S. Dhami and J. B. Stothers. Can. J. Chem. 43, 510 (1965); (c) E. Sol'aniova, S. Toma, and S. Gronowitz. Org. Magn. Reson. 8, 439 (1976).

11. J. A. Pople. Proc. R. Soc. London, A239, 550 (1957).

12. G. J. Ray, R. J. Kurland, and A. K. Colter. Tetrahedron, 27, 735 (1971).

13. R. Hagen and J. D. Roberts. J. Am. Chem. Soc. 91, 4504 (1969).

14. W. F. Reynolds and G. K. Hamer. J. Am. Chem. Soc. 98 , 7296(1976).

15. W. D. AdCock, B. D. GuPTA, and W. Kitching. J. Org. Chem. 41, 1498(1976). 